\title{
INTERNATIONALER ZUSAMMENSCHLUSS IN DER WISSENSCHAFTLICHEN TIERGÄRTNEREI
}

\author{
VON \\ Dr. KURT PRIEMEL \\ Präsident des Internationalen Verbandes der Direktoren Zoologischer Gärten \\ Garmisch
}

Wenn die Internationale Organisation der Direktoren der grossen, wissenschaftlich geleiteten Tiergärten ihre 50. Jahrestagung mit der HundertjahrFeier der Königlichen Zoologischen Gesellschaft Natura Artis Magistra in Amsterdam im Mai 1938 zusammenfallen lässt, so will sie damit bekunden, wie sehr verbunden sie sich mit dem alt-ehrwürdigen Amsterdamer Zoologischen Garten fühlt und wie hoch sie die Bedeutung dieses Instituts für. die biologischen Wissenschaften und für das Kulturleben der Niederlande zu schätzen weiss. Das gemeinsame Jubiläum der beiden Organisationen scheint Anlass genug, in der Festschrift der „Artis” eine kurze Betrachtung über das Entstehen, den Zweck und die Ziele des massgebenden Tiergärtnerverbandes anzustellen.

In den Jahrzehnten vor dem Weltkriege fanden alljährlich im Frühjahr im Antwerpener Zoologischen Garten Tierversteigerungen statt, welche die „Société Royale de Zoologie d'Anvers" veranstaltete. Diese gaben Gelegenheit zum Zusammentreffen der Leiter der meisten grossen Zoologischen Gärten Europas und erfüllten so, neben ihrem ursprünglichen und eigentlichen Handelszweck, gleichzeitig die Aufgahe nutzbringenden Erfahrungsaustausches. Dabei entstand der Wunsch nach einer jährlichen Zusammenkunft der deutschen Tiergartenleiter, wechselnd zwischen den grossen Gärten innerhalb des Reichsgebietes. Dieser Wunsch wurde 1887 erstmalig verwirklicht. Die so geschaffene zwanglose „Konferenz der Direktoren deutscher Zoologischer Gärten” tagte mit Ausnahme der beiden Kriegsjahre 1915 und 1917, in der Regel in jedem Herbst, vermittelte den leitenden Tiergartenfachleuten die Kenntnis der grossen Tierschau-Institute, insbesondere der entstehenden Neuanlagen, gab 
Gelegenheit zur Behandlung aller Fachfragen und förderte die Kollegialität innerhalb des kleinen, so besonders gearteten Berufskreises.

Da nach dem Weltkriege keine Tierversteigerungen in Antwerpen mehr stattfanden, war für diese lose Vereinigung ein Erfahrungsausiausch mit den Fachleuten ausserhalb der deutschen Reichsgrenzen sehr erschwert. So entstand 1923 der Wunsch nach einer internationalen Verbreiterung der allherbstlichen Zusammenkünfte und es fanden sich zunächst die Tiergartenleiter der deutschsprachigen Länder Oesterreich mit Wien-Schönbrunn und die Schweiz mit Basel zu dieser losen „Konferenz”, dann allmählich die der anderssprachigen Länder, welche die damals noch allein-giltige deutsche Verhandlungssprache beherrschten. Wenige Jahre später waren auch schon die Gärten Amsterdam, Antwerpen, Budapest, Kopenhagen, Posen, Rotterdam, Stockholm und Warschau in dem losen Verband vertreten, der inzwischen den Namen „Konferenz der Direktoren Mitteleuropäischer Zoologischer Gärten” angenommen hatte.

Tiergarten-Neugründungen und Ausbau bestehender älterer Gärten in den Nachbarländern, sowie das Bedürfnis einer fachlichen Fühlungnahme mit den Leitern der bedeutendsten Tiergärtnern in England und Amerika liessen eine weitere Vergrösserung der Vereinigung auf internationaler Grundlage gegeben erscheinen. Die in der Zeit von 23- bis 26-9-1935 im Basel tagende 47. „Konferenz”, beschloss daher, eine Umorganisation und begründete damit den „Internationalen Verband der Direktoren Zoologischer Gärten."

Die Aufgaben der neuen. Vereinigung blieben die gleichen wie bisher. Die Mitgliedschaft ist auch im neusgestalteten Verband eine ,persönliche”, - die einzelnen Instituie werden also durch die Person ihrer Leiter in Verband vertreten. Die Aufnahme kann nicht beantragi werden, sondern die neuen Mitglieder werden, nachdem sie vom Präsidenten vorgeschlagen und mit mindestens $4 / 5$. Mehrheit aufgenommen sind, von diesem berufen. Voraussetzung ist, dass die zur Mitgliedschaft vorgeschlagenen im Fachgebiet an leitender Stelle und nach wissenschaftlichen Grundsätzen praktisch tätig sind.

Verhandlungssprachen sind: Deutsch, Englisch und Französisch. Der Verbandspräsident wird durch Stimmenmehrheit in geheimer Abstimmung auf 4 Jahre gewählt. Ihm liegt der gesamte Schriftwechsel und die Ventretung des Verbandes nach aussen ob. Verbandsorgan ist die Zeitschrift: „Der Zoologische Garten", welche 1859 vom Zoologischen Garten Frankfurt am Main, bezw. der dortigen „Zoologischen Gesellschaft” gegründet und im November I888, laut Beschluss der „Direktoren-Konferenz”, zum „Organ sämtlicher Zoologischer Gärten Deutschlands" erklänt wurde.

Eine Erweiterung der Aufgahen des Verbandes ist dadurch gegeben, dass er, ausser denen der Zoologischen Gärten, auch die gemeinsamen Bestrebungen der Naturschutzparks und der gleichgerichteten Tierliebhaberei und Tierzucht zu fördern sucht. Für die immer vordringlicher werdenden Aufgaben des Naturschutzes, - teils unmittelbar, teils werbend -, sich einzusetzen, liessen sich schon im Laufe der letzten 3 Jahrzehnte manche Zoologischen Gärten als 
Ehrenpflicht angelegen sein. Da viele der dringendsten Forderungen des Naturschutzes internationaler Art sind, hofft der Verband in seiner helitigen Form auch auf diesem Gebiet Wesentliches leisten zu können.

Die Einrichtung des Verbandes hat sich in jeder Hinsicht bewährt. Der durch ihn erreichte kollegiale Kontakt vieler massgebender Tiergartenfachleute hat schon manche Früchte getragen. Gerade im tiergärtnerischen Beruf, der neben seinen wissenschaftlich-biologischen Grundlagen auch technische Kenntnisse und Erfahrungen, Fähigkeiten im Veranstaltungswesen, kaufmännischen Sinn und Verwaltungseignung erfordert, ist eine ständige Fühlung der führenden Vertreter in verschiedenen Ländern und ein fortlaufender Erfahrungsaustausch unter ihnen besonders wünschenswert. Der Verband, welcher sich bis jetzt über Io verschiedene Staaten, nämlich Belgien, Dänemark, Deutschland, Frankreich, Grossbritannien, Holland, Polen, Schweiz, Ungarn und die Vereinigten Staaten von Amerika erstreckt, sucht diesen Forderungen nachzukommen, ausser durch den Schriftwechsel der Verbandsleitung mit den Mitgliedern, durch die alljährlichen $4-5$ tägigen Zusammenkünfte. Diese finden wechselnd in den verschiedenen Instituten der Mitglieder, vor allem in solchen, die Neues zu bicien haben, statt. Vorträge, teils mit Lichtbildern und Filmen, Vorweisungen, gründliches Studium des gastgebenden Gartens, vor allem seiner Neuanlagen unter Führung des Leiters und seiner wichtigsten Mitarbeiter, Besichtigungen verwandter und einschlägiger Betriebe und Einrichtungen im Umkreis der Tagungsorte, Vermittelung der Kenntnis der betreffenden Städte und nicht zuletzt Fühlungnahme mit den Verwaltungskörperschaften des gastgebenden Gartens und mit den leitenden Männern der ihn fördernden Behörden sind die wesentlichsten Momente der Veranstaltungsfolgen. Dazu kommt eine schöne und sehr bewährte Pflege der Geselligkeit unter den Tiergartenfachleuten und mit Vertretern der verwandten Wissenschaften und der tierdarstellenden bildenden Künste.

Die Haltung lebender Tiere für Schauzwecke ist bisher leider kein Privileg der dazu Berufenen bzw. der dafür Geeigneten. Das hat dem Ansehen der Sache der Tiergärten nicht wenig geschadet. Es ist tief bedauerlich, dass heut roch Einrichtungen, die teils aus Sensationsmache, teils aus falsch verstandener Tierliebe, teils lediglich aus gewinnsühtigen Zwecken entstanden sind, alle aber mehr oder minder in Folge von mangelnder Sachkenntnis oder von Vernachlässigung durch das Fehlen eines soliden finanziellen Unterbaues, sich gegen die Grundforderungen eines vernunftgemässen Tierschutzes dauernd vergehen, als „Tiergärten” sich bezeichnen können. Auch unter dem Begriff der sog. „Heimattiergärten” verbirgt sich in vielen Fällen mancherlei Unerfreuliches, teils auch Schädliches. In Deutschland ist man dabei, geschmackverderbenden und tierquälerischen Tierhaltungseinrichtungen ein Ende zu bereiten. Aber auch so manche anerkannte Zoologischen Gärten oder Tiergärten sind noch weit davon entfernt, ihre volksbildenden, wissenschaftlichen und naturschützerischen Aufgaben erkannt zu haben, geschweige denn ihnen nachzu- 
kommen. Dem Verband, der, wie schon gesagt, nur Leiter wissenschaftlich geführter Gärten vereinigt, erwächst die Aufgabe, ganz allgemein für die Wahrung eines wissenschaftlichen und ästhetisch befriedigenden Standards der öffentlichen Haltungen lebender Tiere hinzuwirken. Als weitere Aufgabe wird für ihn die Notwendigkeit unabweisbar werden, auf das Heranziehen eines guten Nachwuchses und dessen Einsetzung zur rechten Zeit an richtiger Stelle bedacht zu sein.

Noch steht der Verband am Anfang seiner Arbeit. Dass diese sich mehr und mehr zum Segen der Sache der Tiergärten auswirken möge, sei unser Wunsch! 